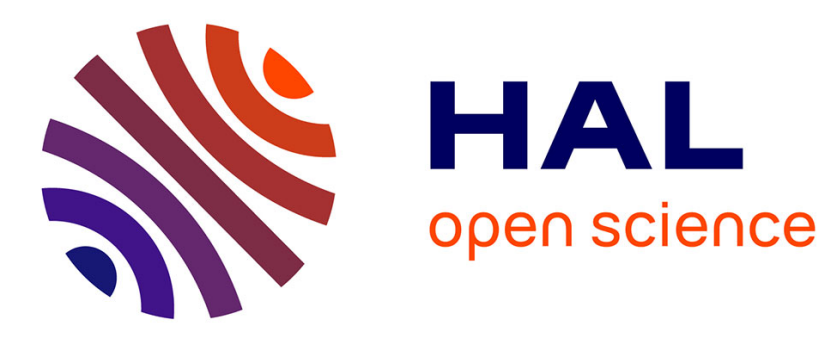

\title{
Modeling Pointing Tasks in Mouse-Based Human-Computer Interactions
}

Stanislav Aranovskiy, Rosane Ushirobira, Denis Efimov, Géry Casiez

\section{To cite this version:}

Stanislav Aranovskiy, Rosane Ushirobira, Denis Efimov, Géry Casiez. Modeling Pointing Tasks in Mouse-Based Human-Computer Interactions. CDC 2016 - 55th IEEE Conference on Decision and Control, Dec 2016, Las Vegas, United States. 10.1109/CDC.2016.7799284 . hal-01380318

\section{HAL Id: hal-01380318 https://hal.inria.fr/hal-01380318}

Submitted on 12 Oct 2016

HAL is a multi-disciplinary open access archive for the deposit and dissemination of scientific research documents, whether they are published or not. The documents may come from teaching and research institutions in France or abroad, or from public or private research centers.
L'archive ouverte pluridisciplinaire HAL, est destinée au dépôt et à la diffusion de documents scientifiques de niveau recherche, publiés ou non, émanant des établissements d'enseignement et de recherche français ou étrangers, des laboratoires publics ou privés. 


\title{
Modeling Pointing Tasks in Mouse-Based Human-Computer Interactions
}

\author{
Stanislav Aranovskiy ${ }^{1}$, Rosane Ushirobira ${ }^{1}$, Denis Efimov ${ }^{1,2}$ and Géry Casiez ${ }^{2,3}$
}

\begin{abstract}
Pointing is a basic gesture performed by any user during human-computer interaction. It consists in covering a distance to select a target via the cursor in a graphical user interface (e.g. a computer mouse movement to select a menu element). In this work, a dynamic model is proposed to describe the cursor motion during the pointing task. The model design is based on experimental data for pointing with a mouse. The obtained model has switched dynamics, which corresponds well to the state of the art accepted in the humancomputer interaction community. The conditions of the model stability are established. The presented model can be further used for the improvement of user performance during pointing tasks.
\end{abstract}

\section{INTRODUCTION}

Pointing is a fundamental task in any graphical user interface requiring users to cover a distance to select a target with a given width. In the case of direct interactions (e.g. on smartphones and tablets), users move their hand and fingers while touching the screen where the target is located. In the case of indirect interactions, users remotely control a pointer displayed on the screen using a computer mouse or a touchpad. Indirect interactions allow to adjust the relationship (transfer function) between the physical displacement of the finger or mouse and the pointer on screen. The transfer function defines a multiplier (Control-Display gain or CD gain) applied on the input device velocity to determine the pointer velocity [1]. The CD gain can be constant or dynamically adjusted over time depending on the input device velocity (dynamic transfer functions). All modern operating systems use dynamic pointing transfer functions (PTFs) having different shapes and whose performances vary accordingly to the target characteristics [2]. The optimization of these functions remains an open question due to the lack of models describing the dynamics of human pointing. Such pointing models would also be relevant in the context of endpoint prediction [3], where the system must know in advance where the user will point at, in order to modify the visual feedback. Dynamically expanding targets in a toolbar is a well-known example of such techniques [4]. Also, some tools and methods to improve $D / W$ ratio including target prediction can be found in [5]. $01)$

*This work was supported by ANR (TurboTouch, ANR-14- CE24-0009-

${ }^{1}$ Inria, Non-A team, 40 avenue Halley, 59650 Villeneuve d'Ascq, France. stanislav.aranovskiydinria.fr

${ }^{2}$ CRIStAL (UMR CNRS 9189), Villeneuve d'Ascq, France.

3 Inria, Mjolnir team, 40 avenue Halley, 59650 Villeneuve d'Ascq, France.

\section{Modeling pointing motions}

Popular models of pointing motion used for interface design and for ergonomics improvement are usually static. They do not describe the exact pointing motion, but consider only averaged static relationships. The most famous and ubiquitous model, Fitts' law, was proposed in the seminal paper [6] and is given by

$$
M T=a+b \log _{2}\left(1+\frac{D}{W}\right),
$$

where $M T$ is the movement time, $a$ and $b$ are positive constants, $W$ is the target width and $D$ is the distance to the target center, and the logarithmic term characterizes difficulty of the task. Since the relation (1) was verified in various experimental studies with different environmental conditions [7], many researchers were motivated to find the reasons behind Fitts' law. To this end, the Iterative Corrections model has been proposed in $[8]$. This model considers the pointing motion as governed entirely by a visual feedback, while the Impulse Variability model proposed by [9] attributes the motion almost completely to an initial muscle impulse. However, it was shown that neither of these models takes into account all the diverse effects observed in experiments, so the hybrid Optimized Initial Impulse model has been developed by Meyer et al. in [10]. This latter is accepted by the Human-Computer Interaction (HCI) community as the most complete and successful explanation for Fitts' law [5]. According to Meyer's model, a pointing motion can be divided into two stages: a rapid and large movement bringing the pointer reasonably close to the target without visual tracking, followed by a slower corrective movement hitting the target under visual feedback control. It is worth stressing that the models in $[8]-[10]$ almost do not contain differential equations for motion description, but mainly determines possible pointing scenarios verbally.

The authors in [11] have observed in experiments that for the rapid motion, called ballistic, the movement time $M T$ depends weakly on the target width $W$ and is mainly proportional to $\sqrt{D}$. They have also noticed that for pointing tasks with low difficulty, i.e. with relatively large values of $W$, the target may be reached by the ballistic motion only.

Nowadays, Fitts' law and the Optimized Initial Impulse model remain the most widely accepted static models of pointing motions. Recent advances in this direction are mainly focused on their extension, e.g. a model for analyzing rapid point-and-click motions taking into account human effects has been recently proposed in [12]. 
On the other hand, dynamic models are not as well studied and are not so numerous as static ones. The Vector Integration To Endpoint (VITE) model [13] was widely applied in robotics to design human-like pointing movements [14]. This model describes a motion governed by an agonist-antagonist pair of muscles, e.g. wrist rotation. For one muscle, the VITE model is given by

$$
\begin{aligned}
& \dot{P}(t)=G(t)[V(t)]^{+}, \\
& \dot{V}(t)=\gamma(-V(t)+T-P(t)),
\end{aligned}
$$

where $T$ is the target position, $P(t)$ is the pointer position, $V(t)$ is the pointer velocity, $\gamma>0$ is a constant, $G(t)$ is the "GO" signal, which gates the movement execution and is often assumed to be a positive constant or a step function, and $[\cdot]^{+}$denotes a positive projection. By considering two equivalent muscles, the projection in (2) can be omitted, then the VITE model yields a linear time-invariant secondorder system with the stable equilibrium point $P=T, V=0$. Moreover, since for linear systems the transient time is logarithmically related with the traveled distance, then VITE model asymptotic behavior reproduces the Fitts' law (1). A slightly modified version of the VITE model with $G(t) \equiv 1$ was used in [15] to study sensorimotor integration in absence of visual feedback.

Very recently, the VITE model has been extended in [16] to handle pointing transfer functions. In this work, the "GO" signal $G$ in (2) has been replaced with a motion acceleration function $G(\|V\|)$ and closed-loop stability has been proven assuming no feedback delay with a non-decreasing $G(\|V\|)$ function.

Nevertheless, the VITE model (2) is capable only to describe the corrective stage of a pointing motion and it is not able to model the ballistic part with movement time proportional to $\sqrt{T}$. Hence, the problem of designing a dynamic model that is able to represent both ballistic and corrective stages remains open.

\section{Problem statement}

The aim of this work is to identify a dynamic model of pointing motion based on experimental data with computer mouse from [2] and using the methods of control systems theory. Motivated by the Optimized Initial Impulse model [10], the obtained model should have hybrid or switching nature to take into account ballistic and visual tracking phases. Additionally, it should consider the PTF implemented in the operating system as in [16] (the case of a static PTF is selected). Such a model can be further used for designing PTFs that improve user performance in pointing tasks.

The outline of this paper is as follows. In Section III we describe the experimental data used in our studies. The proposed model and investigation of its properties are given in Section III. In Section IV] we present the results of experimental validation, and the paper is wrapped up with conclusions and discussion of future directions in Section $\mathrm{V}$.

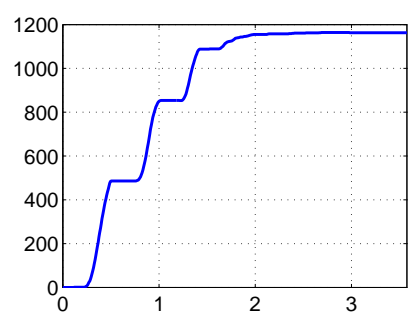

(a) Non-adapted.

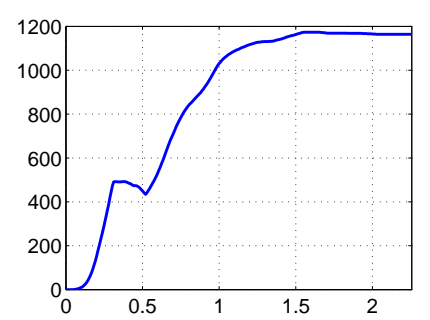

(b) Partially adapted.

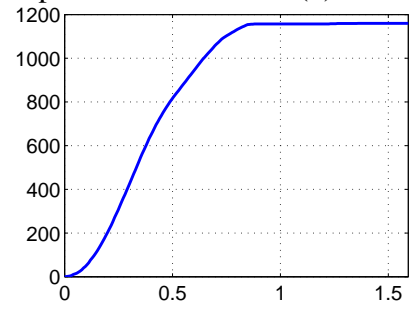

(c) Adapted.

Fig. 1: Pointing trajectories for participants differently adapted to a constant-gain PTF, cursor position (in pixels) versus time (in seconds).

\section{EXPERIMENTAL DATA DESCRIPTION}

The model design is based on the data from the experiments described in [2], where the authors compared the performance of default transfer functions used by modern operating systems (Windows, OS X and Xorg) in a one dimensional pointing task. They also added a constant CD gain function, as a baseline for comparison. Participants used a 400 counts-per-inch USB corded Logitech mouse to select targets displayed on a 23" display. Targets were rendered as solid vertical bars 1163 pixels $(300 \mathrm{~mm})$ apart with 4 target widths corresponding to 9 pixels $(2.32 \mathrm{~mm}), 6$ pixels $(1.55 \mathrm{~mm}), 3$ pixels $(0.77 \mathrm{~mm})$ and 1 pixel $(0.26 \mathrm{~mm})$. All the details about the experimental setup and procedure are available in [2].

Below, we keep only the data for the constant gain PTF. Such a choice eliminates all the uncertainties related to PTFs with variable gains and allows us to focus on modeling of human movements. However, it should be highlighted that such a constant PTF is not of everyday use for most participants [2]. This fact is especially important during the ballistic movement phase, which is not visually tracked: some participants performed usual wrist-elbow gestures expecting to find the cursor reasonably close to the target position, but they did not. Typically, a constant PTF provides less motion acceleration than a PTF with variable gain, thus the cursor is found farther from the target than expected. If a participant did not adapt his/her gestures to the constant PTF, then several ballistic motions had been performed before the corrective phase began. In Fig. 1 trajectories of adapted (trained), partially adapted, and non-adapted participants are given illustrating multiple ballistic phases. Further we will use only data associated with the adapted participants. 


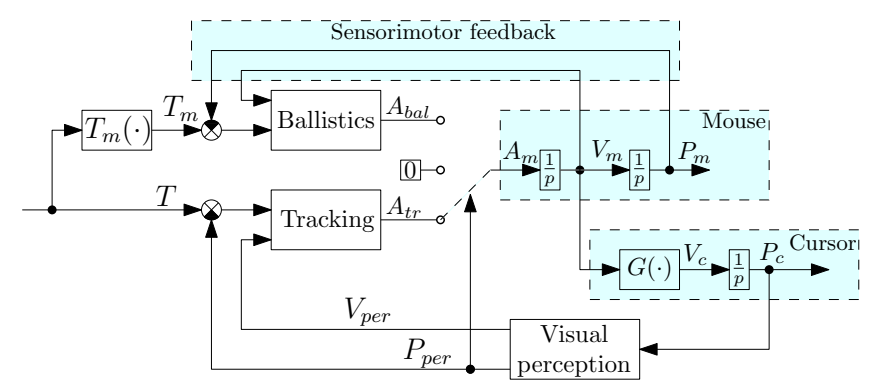

Fig. 2: The structure of the proposed pointing movement model.

\section{MODEL}

\section{A. Model overview}

The structure of the proposed model is given in Fig. 2 The dynamics of the pointing device, in our case a mouse, is described by the double integrator:

$$
\begin{aligned}
& \dot{P}_{m}(t)=V_{m}(t), \\
& \dot{V}_{m}(t)=A_{m}(t),
\end{aligned}
$$

where $P_{m}(t)$ is the mouse position, $V_{m}(t)$ is the mouse velocity, and $A_{m}(t)$ is the mouse acceleration. The mouse velocity is measured in counts per second and it is mapped to the cursor velocity $V_{c}(t)$ (measured in pixels per second), with the PTF $G(\cdot)$ given by:

$$
V_{c}=G\left(V_{m}\right) .
$$

The model under consideration has a constant gain PTF, thus

$$
G\left(V_{m}\right):=g_{0} V_{m},
$$

where $g_{0}>0$ is a constant representing both velocity amplification and counts-to-pixels scaling.

The cursor position $P_{c}(t)$ satisfies the following differential equation

$$
\dot{P}_{c}(t)=V_{c}(t)
$$

and it is visually perceived yielding the observed cursor position $P_{\text {per }}(t)$ and the observed cursor velocity $V_{\text {per }}(t)$. The perception is modeled with the first-order LTI stable filter

$$
\begin{aligned}
& V_{\text {per }}(t)=\frac{1}{\tau_{p e r}}\left(P_{c}(t)-P_{\text {per }}(t)\right), \\
& \dot{P}_{\text {per }}(t)=V_{\text {per }}(t),
\end{aligned}
$$

which can also be written as

$$
\begin{aligned}
& P_{\text {per }}(t)=\frac{1}{\tau_{\text {per }} p+1} P_{c}(t), \\
& V_{\text {per }}(t)=\frac{p}{\tau_{p e r} p+1} P_{c}(t),
\end{aligned}
$$

where $p:=\frac{d}{d t}$ and $\tau_{p e r}>0$ is the perception time constant.

The perceived position $P_{\text {per }}(t)$ is used as a switching signal for the mouse acceleration $A_{m}(t)$. First, when the pointing motion has just begun, the movement is governed by the ballistic dynamics. Next, when the participant visually marks the cursor, which means the cursor has reached some predefined position $P_{s w}$, the motion switches to the visual tracking. This commutation is not instantaneous, and during the commutation period $\delta_{s w}>0$ zero acceleration is applied. Let $t_{s w}>0$ be the first instant of time when $P_{p e r}(t)=P_{s w}$. Then

$$
A_{m}(t)= \begin{cases}A_{b a l}(t) & \text { for } t<t_{s w} \\ 0 & \text { for } t_{s w} \leq t \leq t_{s w}+\delta_{s w} \\ A_{t r}(t) & \text { for } t_{s w}+\delta_{s w}<t\end{cases}
$$

where $A_{b a l}(t)$ and $A_{t r}(t)$ are the accelerations provided by the ballistic dynamics and the tracking dynamics correspondingly.

The reference signal $T$ is the desired cursor position measured in pixels and is assumed to be a constant or a step function. When the value of $T$ is set, the participant estimates the desired mouse displacement $T_{m}=T_{m}(T)$, which is measured in $\mathrm{mm}$, according to his/her experience with the used PTF. We assume that this "aiming function" can be approximated well as a constant gain,

$$
T_{m}(T):=g_{m} T
$$

where $g_{m}>0$.

The value $T_{m}$ initiates the ballistic movement that is supposed to bring the cursor sufficiently close to the desired position $T$. However, if the user is not well-experienced with the given PTF, then the resulting ballistic movement may have poor accuracy, see Fig. 1a

Now we are going to provide the models of the accelerations $A_{t r}$ and $A_{b a l}$, which correspond to the tracking and ballistic phases, respectively.

Remark 1: It is worth noting that for the considered scenario there is no stability issue related to switching, since for adapted users each phase is activated just once.

\section{B. Tracking dynamics}

The tracking dynamics is an extension of the VITE model (2), (3) that takes into account the visual perception (5) with

$$
A_{t r}(t):=\gamma\left(-k_{v} V_{p e r}(t)+T-P_{p e r}(t)\right),
$$

where $k_{v}>0$ is a model parameter. Considering the model in the corrective stage, i.e. for $t>t_{s w}+\delta_{s w}$, and combining together the mouse and cursor dynamics, the perception and the tracking law (7), we result with the following closed-loop system:

$$
\begin{aligned}
\dot{P}_{p e r}(t) & =\frac{1}{\tau_{p e r}}\left(P_{c}(t)-P_{p e r}(t)\right), \\
\dot{P}_{c}(t) & =V_{c}(t), \\
\dot{V}_{c}(t) & =g_{0} \gamma\left(-\frac{k_{v}}{\tau_{p e r}}\left(P_{c}(t)-P_{p e r}(t)\right)+T-P_{p e r}(t)\right) .
\end{aligned}
$$

Proposition 1: Consider the system (8) with $\gamma>0, g_{0}>0$, and $k_{v}>\tau_{\text {per }}>0$, then the system is exponentially stable with the equilibrium point $P_{p e r}=P_{c}=T$ and $V_{c}=0$.

The proof of Proposition 1 is omitted due to the lack of space. 


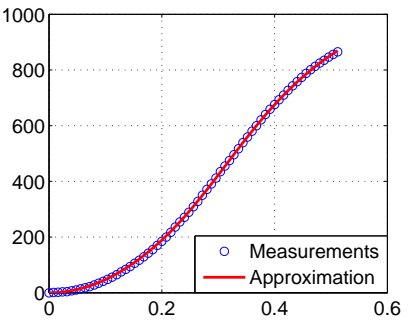

(a) Position (in pixels) versus time (in seconds).

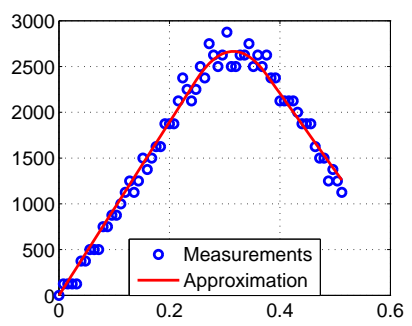

(b) Velocity (in pixels per second) versus time (in seconds).

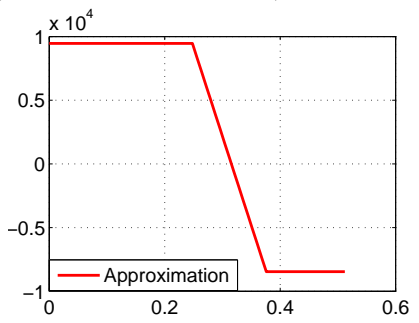

(c) Acceleration (in pixels per square seconds) versus time (in seconds).

Fig. 3: Measured mouse position and velocity for an experienced participant and their approximations.

\section{Ballistics}

The ballistic movement is performed without visual feedback, i.e. users do not observe the cursor position during this phase. However, due to sensorimotor integration and proprioception, users can estimate their wrist velocity and displacement yielding the mouse velocity and position feedback, see Fig. 2 .

Analysis of the experimental data described in Section II shows that a typical ballistic movement can be approximated well with the curve presented in Fig. 3, where both measurements and approximations are given. The approximating curve is defined by its acceleration profile, Fig. 3c that consists of two constant levels with a linear transient between them. It is worth noting that this ballistic movement is not finished, i.e. the mouse velocity did not reach zero and the participant's wrist did not stop. The reason is that, as it was found from the data, experienced participants usually notice the cursor and switch to visual tracking before finishing the ballistic phase, $c f$. Fig. $1 \mathrm{a}$ and Fig. 1c.

The ballistic movement model is proposed in the form

$$
\begin{aligned}
& \dot{\tilde{P}}_{m}(t)=V_{m}(t) \\
& \dot{V}_{m}(t)=A_{b a l}(t)=f_{b a l}\left(\tilde{P}_{m}(t), V_{m}(t)\right)
\end{aligned}
$$

where $\tilde{P}_{m}(t):=P_{m}(t)-T_{m}$, and the function $f_{b a l}(\cdot, \cdot)$ is to be defined.

To calculate the function $f_{b a l}$, we define the acceleration profile, Fig. $3 \mathrm{c}$, for the initial conditions $\tilde{P}_{m}(0)=-T_{m}$ and
$V_{m}(0)=0$ as

$$
A_{b a l}(t)= \begin{cases}a_{1} & \text { for } t<t_{1}, \\ a_{1}+l\left(t-t_{1}\right) & \text { for } t_{1} \leq t \leq t_{2}, \\ a_{2} & \text { for } t>t_{2},\end{cases}
$$

where $a_{1}, a_{2}$ and $t_{2}>t_{1}>0$ are profile parameters, $a_{1} a_{2}<0$, and

$$
l:=\frac{a_{2}-a_{1}}{t_{2}-t_{1}}
$$

is the slope of the linear part, $a_{1} l<0$. Define also

$$
\eta(t):=-\tilde{P}_{m}(t)-k_{b a l} V_{m}(t),
$$

where $k_{b a l}>0$ is a ballistic model parameter. With some tiresome but straightforward computations, it can be shown that the choice

$$
k_{b a l}=\frac{2 a_{1}^{3}-6 a_{1}^{2} l t_{1}+3 a_{1} l^{2} t_{1}^{2}-6 l^{2} T_{m}}{3 a_{1} l\left(a_{1}-2 l t_{1}\right)}
$$

ensures that for $t \in\left[t_{1}, t_{2}\right]$ the following holds:

$$
\eta(t)=A_{b a l}(t)\left(p_{2} A_{b a l}^{2}(t)+p_{1} A_{b a l}(t)+p_{0}\right),
$$

where

$$
p_{0}:=\frac{a_{1}\left(a_{1}-2 l t_{1}\right)}{2 l^{2}}, \quad p_{1}:=-\frac{k_{b a l}}{2 l}, \quad p_{2}:=-\frac{1}{6 l^{2}},
$$

and $p_{0}>0, p_{2}<0$. Obviously, there is a value of the gain $g_{m}$, defined in (6), such that $k_{b a l}$ computed in (11) is positive.

To construct the function $f_{b a l}\left(\tilde{P}_{m}, V_{m}\right)$ we want to inverse (12) on the interval $\left[a_{l}, a_{r}\right]$, where $a_{l}:=\min \left(a_{1}, a_{2}\right)<0$ and $a_{r}:=\max \left(a_{1}, a_{2}\right)>0$. Define such an inversion as $A_{b a l}=$ $\phi(\eta)$, and denote

$$
\begin{aligned}
& \eta_{l}:=a_{l}\left(p_{2} a_{l}^{2}+p_{1} a_{l}+p_{0}\right), \\
& \eta_{r}:=a_{r}\left(p_{2} a_{r}^{2}+p_{1} a_{r}+p_{0}\right) .
\end{aligned}
$$

To proceed we need the following assumption.

Assumption 1: The parameters $t_{1}, a_{1}, a_{2}$ and $l$ are such that the inequality

$$
t_{1}>\frac{a_{1}^{2}-a_{2}^{2}}{2 a_{1} l} .
$$

holds.

Now we can formulate the following proposition, whose proof is omitted due to the lack of space.

Proposition 2: Choose $0<k_{\text {bal }}<\bar{k}$, where

$$
\bar{k}:=\frac{a_{1}^{2}-a_{2}^{2}-2 t_{1} a_{1} l}{2 a_{2} l},
$$

where $\bar{k}>0$ due to Assumption 1 Then $\phi(\eta)$ for $\eta \in\left[\eta_{l}, \eta_{r}\right]$ is a uniquely defined real-valued monotonic function with $\phi\left(\eta_{l}\right)=a_{l}, \phi(0)=0$ and $\phi\left(\eta_{r}\right)=a_{r}$.

Remark 2: Actually, Assumption 1 is not restrictive. It implies that the velocity does not cross zero during the linear part of the acceleration profile. This assumption is reasonable for any practical ballistic movement, see Fig. 3. Particularly, it is satisfied for all the experimental data we analyzed.

Remark 3: The inequality $k_{b a l}<\bar{k}$ also restricts admissible values of $T_{m}$, see (11). In other words, there exists the upper bound $\bar{g}_{m}$, such that $k_{b a l}<\bar{k}$ is satisfied only for 


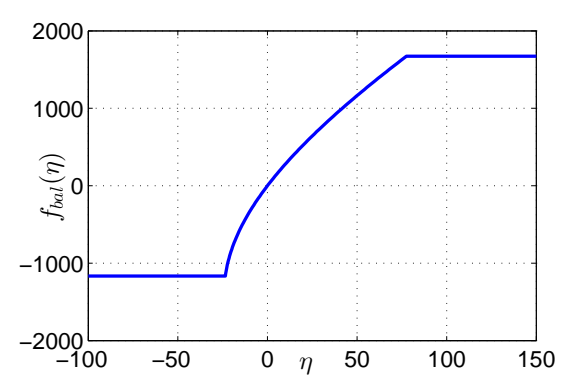

Fig. 4: An example of the function $f_{b a l}(\eta)$ reconstructed from the experimental data.

$g_{m}<\bar{g}_{m}$, where $g_{m}$ is defined in (6). Practically it means that given the parameters $a_{1}, t_{1}$, and $l$, the ballistic movement cannot achieve arbitrary big values $T_{m}$.

Now we are in the position to construct the desired function $f_{b a l}\left(\tilde{P}_{m}, V_{m}\right)$. Using definition (10) and choosing $k_{b a l}, \eta_{l}, \eta_{r}$ and $\phi(\eta)$ according to Proposition 2, we define

$$
f_{\text {bal }}(\eta):= \begin{cases}a_{2} & \text { for } \eta<\eta_{l}, \\ \phi(\eta) & \text { for } \eta_{l} \leq \eta \leq \eta_{r} \\ a_{1} & \text { for } \eta>\eta_{r}\end{cases}
$$

It is worth noting that $f_{\text {bal }}(\eta)$ is continuous non-decreasing function, which is strictly monotonic on $\left[\eta_{l}, \eta_{r}\right]$, and $f_{\text {bal }}(0)=0$. An example of the function $f_{\text {bal }}(\eta)$ reconstructed from our experimental data is given in Fig. 4.

To summarize, the ballistic movement model is given by (9), (10) and 13). Stability of this model is considered in the following proposition.

Proposition 3: Consider the system (9), (10) and (13) with $0<k_{b a l}<\bar{k}$. All the trajectories of the system are bounded, and the equilibrium point $\tilde{P}_{m}=V_{m}=0$ is globally asymptotically stable.

The proof of Proposition 3 is omitted due to the lack of space.

\section{EXPERIMENTAL VALIDATION}

In this section the results of experimental verification of the proposed model are presented. From the data set described in Section [I] we have chosen three trajectories corresponding to an adapted (trained) participant with a constant-gain PTF. The mouse had 400 counts per inch, and the display admitted a resolution 98.5 pixels per inch. The gain of the used PTF equals

$$
g_{0}=1.5 \frac{98.5}{400}
$$

and it provides both counts-to-pixels scaling and 1.5 times velocity amplification. The sampling frequency is $125 \mathrm{~Hz}$.

For each of these trajectories we have identified parameters of the model; see Table I for the list. Comparison of the measured data with the model output is presented in Figs. 5, 6 and 7 Here the first trajectory corresponds to a typical pointing movement, the second trajectory presents a movement with an overshoot due to a long ballistic motion,
TABLE I: List of the model parameters.

\begin{tabular}{|c|c|}
\hline Ballistics & $a_{1}, a_{2}, t_{1}, t_{2}, g_{m}, k_{b a l}$ \\
\hline Switching & $P_{s w}, \delta_{s w}$ \\
\hline Tracking & $\tau_{p e r}, k_{v}, \gamma$ \\
\hline
\end{tabular}

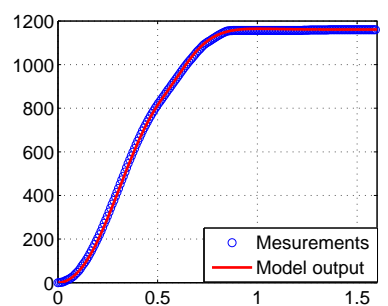

(a) Position (in pixels) versus time (in seconds).

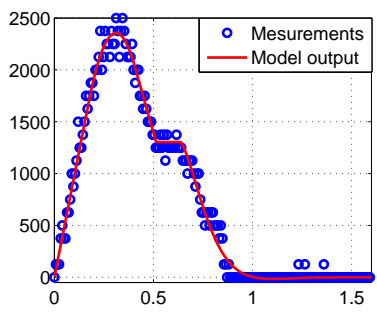

(b) Velocity (in pixels per second) versus time (in seconds).
Fig. 5: Comparison of the measurements with the model outputs, trajectory \#1.

and the third trajectory has a relatively short ballistic phase. As it can be seen from the figures, the proposed model can handle all these types of pointing motions and fits well the measurements.

\section{CONCLUSIONS}

The problem of modeling a pointing movement with a computer mouse has been considered in this paper. Following the Optimized Initial Impulse model [10], we have divided the pointing motion into three phases: the ballistics phase, the commutation phase with zero acceleration and the tracking. Therefore, the proposed model is a switching model. The ballistic phase is not visually guided by the user, but it has a sensorimotor feedback and it is modeled by a nonlinear system of Lurie form. When the predefined cursor position is reached, and the cursor is observed by a user, the model switches after the commutation transients to the tracking phase. In this phase, the user visually perceives the cursor position (the perception is modeled with a linear filter) and the tracking dynamics is given by an extended VITE model [13]. It is shown that both ballistic and tracking dynamics are globally asymptotically stable under some established mild conditions. The problem of instability caused by commutation has not been analyzed since for adapted users, which

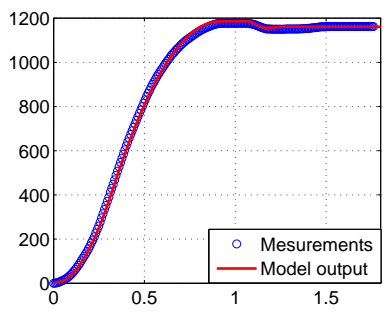

(a) Position (in pixels) versus time (in seconds).

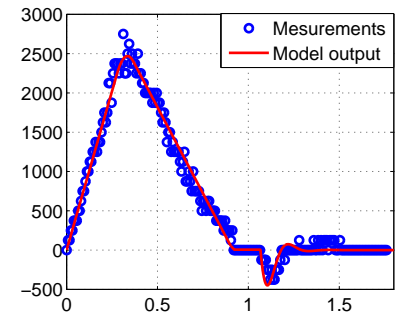

(b) Velocity (in pixels per second) versus time (in seconds).
Fig. 6: Comparison of the measurements with the model outputs, trajectory \#2. 


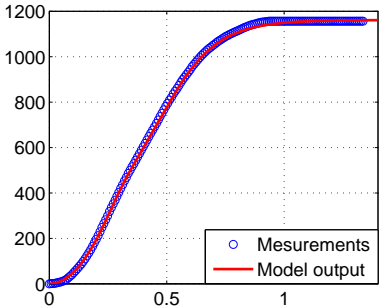

(a) Position (in pixels) versus time (in seconds).

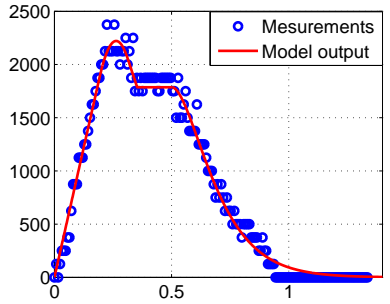

(b) Velocity (in pixels per second) versus time (in seconds).
Fig. 7: Comparison of the measurements with the model outputs, trajectory \#3.

are the only considered in this note, each phase is activated just once.

The model is validated in a series of experiments. Validation results show that the model fits well different types of pointing movements.

Our further researches aim in the following directions. First, the stability analysis and experimental validation have been performed for constant-gain PTFs only. A natural extension is to consider more realistic PTFs that depend both on cursor velocity and position. Second, a model extension for non-adapted users can be obtained, which requires the analysis of stability in the presence of switching (since in such a case the ballistic and the commutation motions can be activated several times before the tracking phase is reached). Third, the effect of delays on the closed-loop stability and the performance of pointing task can be analyzed. Fourth, the proposed model can be applied to identify users in terms of their experience and to tune automatically the interface to match the exact user. A long-term goal in this direction is to propose an optimal (in a certain sense) PTF design given the model, and to extend the approach for different pointing devices.

\section{REFERENCES}

[1] G. Casiez, D. Vogel, R. Balakrishnan, and A. Cockburn, "The impact of control-display gain on user performance in pointing tasks," HumanComputer Interaction, vol. 23, no. 3, pp. 215-250, 2008.
[2] G. Casiez and N. Roussel, "No more bricolage! Methods and Tools to Characterize, Replicate and Compare Pointing Transfer Functions,' Proceedings of the 24th annual ACM symposium on User interface software and technology - UIST '11, p. 603, 2011.

[3] E. Lank, Y.-C. N. Cheng, and J. Ruiz, "Endpoint prediction using motion kinematics," Proceedings of the SIGCHI conference on Human factors in computing systems CHI 07, pp. 637-646, 2007.

[4] M. McGuffin and R. Balakrishnan, "Acquisition of expanding targets," in Proceedings of the SIGCHI Conference on Human Factors in Computing Systems, ser. CHI '02. New York, NY, USA: ACM, 2002, pp. 57-64.

[5] R. Balakrishnan, "'Beating" Fitts' law: Virtual enhancements for pointing facilitation," International Journal of Human Computer Studies, vol. 61, no. 6, pp. 857-874, 2004.

[6] P. M. Fitts, "The information capacity of the human motor system in controlling the amplitude of movement." Journal of Experimental Psychology, vol. 47, no. 6, pp. 381-391, 1954.

[7] I. S. MacKenzie, "Fitts' law as a research and design tool in humancomputer interaction," Human-Computer Interaction, vol. 7, no. 1, p. $48,1992$.

[8] E. R. Crossman and P. J. Goodeve, "Feedback control of handmovement and Fitts' Law." The Quarterly journal of experimental psychology. A, Human experimental psychology, vol. 35, no. Pt 2, pp. 251-278, 1983.

[9] R. a. Schmidt, H. Zelaznik, B. Hawkins, J. S. Frank, and J. T. Quinn, "Motor-output variability: a theory for the accuracy of rapid motor acts." Psychological review, vol. 47, no. 5, pp. 415-451, 1979.

[10] D. E. Meyer, R. a. Abrams, S. Kornblum, C. E. Wright, and J. E. Smith, "Optimality in human motor performance: ideal control of rapid aimed movements." Psychological review, vol. 95, no. 3, pp. 340-370, 1988.

[11] K. C. Gan and E. R. Hoffmann, "Geometrical conditions for ballistic and visually controlled movements." Ergonomics, vol. 31, no. 5, pp. 829-39, 1988.

[12] A. Almanji, A. R. Payne, R. Amor, and T. C. Davies, "A Nonlinear Model for Mouse Pointing Task Movement Time Analysis Based on Both System and Human Effects," IEEE Transactions on Neural Systems and Rehabilitation Engineering, vol. 23, no. 6, pp. 1003-1011, 2015.

[13] D. Bullock and S. Grossberg, "Neural dynamics of planned arm movements: emergent invariants and speed-accuracy properties during trajectory formation." Psychological review, vol. 95, no. 1, pp. 49-90, 1988.

[14] M. Hersch and A. G. Billard, "A biologically-inspired controller for reaching movements," Proceedings of the First IEEE/RAS-EMBS International Conference on Biomedical Robotics and Biomechatronics, 2006, BioRob 2006, vol. 2006, pp. 1067-1072, 2006.

[15] D. M. Wolpert, Z. Ghahramani, and M. I. Jordan, "An internal model for sensorimotor integration," Science, vol. 269, no. 5232, p. 1880 , 1995.

[16] P. Varnell and F. Zhang, "Characteristics of Human Pointing Motions with Acceleration," in 54th Conference on Decision and Control, Osaka, Japan, 2015, pp. 5364-5369. 